\title{
Effects of Swimming Exercise on Circadian Sleep-waking Rhythms and Brain Serotonin Metabolism in Rats
}

\author{
Ayako SUDo, Heihachiro ARITO and Kazuo FUKUDA \\ National Institute of Industrial Health, \\ 21-1, Nagao 6-chome, Tama-ku, Kawasaki, 214 Japan
}

(Received May 12, 1984 and in revised form June 11, 1984)

\begin{abstract}
Polygraphic recordings of circadian sleep-waking rhythms of rats .were made before and after swimming exercise which was loaded to the animals in the dark period. By visual inspection of the recordings, an exercise-induced increase in the amount of slow-wave sleep (SWS) was observed in the next dark period. In the dark period, 15 hours after the swimming, tryptophan (TRP), serotonin and 5-hydroxyindoleacetic acid (5-HIAA) in several regions of rat brain were measured to clarify the mechanisms of the exercise-induced increase in SWS. The concentrations of TRP and 5-HIAA in the midbrain of the exercised rats were significantly higher than in the control ones. The cortical level of 5-HIAA was also high in the exercise group. The serotonin levels in both the brain regions of the exercised rats were nearly the same with the control. From these results, it is inferred that the increased amount of SWS after the swimming exercise might be attributable to enhanced activity of serotonergic neurons in the midbrain and the cerebral cortex.
\end{abstract}

Key words: Swimming-Exercise-Circadian sleep rhythm-SWS-PS-Brain serotonin-Rat

\section{INTRODUCTON}

Recently, much interest has been focused on effects of physical exercise on subsequent sleep. J. Matsumoto ${ }^{1)}$ found that the time of first appearance of slow-wave sleep (SWS) in rats was significantly earlier after treadmill exercise than the control, while the latency of paradoxical sleep (PS) was delayed. Hobson $^{2)}$ reported reduced latency of SWS onset and a subsequent increase in the amount of SWS after moderate treadmill exercise in cats. K. Matsumoto et al. ${ }^{3}$ reported that the amount of deep SWS was significantly increased after 3-hour swimming in rats, whereas the amount of PS was decreased. The increase of SWS after exercise such as marathon was also observed in human subjects. ${ }^{4,5)}$

According to Jouvet, ${ }^{6)}$ a central serotonergic system consisting mainly of the raphe nuclei is presumably involved in induction and maintenance of SWS, and SWS seems to be associated with enhanced turnover of brain serotonin. Therefore, it would be expected that exercise produces changes in brain serotonin turnover 
associated with an increase in SWS. However, little is known about brain serotonin metabolism at the time of appearance of exercise-induced increase of SWS. The present study was designed to investigate the effects of swimming exercise on subsequent sleep-waking rhythms, especially on SWS, and brain serotonin turnover in rats.

\section{Materials ANd Methods}

\section{Animals}

Male SD rats were purchased at 4 weeks of age from Nippon CLEA INC., and kept on 12-hour light-dark cycles with lights-on at 8:30 for 4 to 7 weeks. The temperature and humidity were controlled at $25 \pm 1{ }^{\circ} \mathrm{C}$ and $55 \pm 5 \%$, respectively. Food and water were given ad libitum.

\section{Polygraphic recording}

Electrodes were implanted into these animals at the age of 8 weeks by the method as described previously. ${ }^{7)}$ A week was allowed for recovery from the surgery. Polygraphic recordings of two EEGs and EMG were made continuously and from these records vigilance states were divided by visual inspection into three stages, SWS, PS and waking as in the previous study. ${ }^{7)}$ The temperature, humidity and lighting conditions of the recording environment were the same as described above.

\section{Exercise}

In a preliminary experiment, it was found that the amount of waking was the greatest in the last four hours of the dark period. Therefore, the swimming exercise was imposed to the animals during this time of day in order to minimize the effects of sleep deprivation during the exercise period.

After baseline recording for 3 or 4 consecutive days (control period), the rats were kept individually in polyethylene buckets, $50 \mathrm{~cm}$ in diameter and $60 \mathrm{~cm}$ deep with the water at the $50 \mathrm{~cm}$ level, for 3.5 hours from 5:00 to $8: 30$ in the dark period.

The temperature of the water was maintained between 28 and $25^{\circ} \mathrm{C}$. After removal from the water, the rats were toweled and the polygraphic recordings were made for the subsequent 3 days.

\section{Power Spectral Analysis}

Abnormality of the EEG was checked by power spectral analysis of the parieto-occipital EEG with a signal processor (Model 7T07, San-ei Sokki Co., Ltd.) and higher paper speed $(10 \mathrm{~mm} / \mathrm{sec})$ recordings of the EEG in some rats. These power spectra were derived from the EEG at regular intervals of $90 \mathrm{sec}$. 
The voltages of the EEG activity were sampled for 256 points at intervals of $10 \mathrm{msec}$.

\section{Measurement of Rectal Temperature}

Before and after the swimming exercise, the rectal temperature of the rats was measured with a thermister thermometer (Model MGAIII-219, Shibaura Electronics Co., Ltd.).

\section{Histological examination}

Six rats were examined for the presence of stomach bleeding by visual inspection after swimming. Two rats loaded with swimming were also checked for stomach ulceration microscopically after Hematoxylin-Eosin staining of the stomach tissue.

\section{Brain Indoleamine Analysis}

For the measurement of brain tryptophan (TRP), serotonin and 5-hydroxyindoleaceitc acid (5-HIAA), 11-week-old rats were loaded with the swimming in the same method as above, and about 15 hours later (23:00-24:00) they were killed by decapitation. The brain of each rat was removed quickly and dissected on an ice plate according to the method of Glowinski and Iversen. ${ }^{8)}$ Cerebral cortex, midbrain, hippocampus, hypothalamus and pons-medulla were stored at $-70^{\circ} \mathrm{C}$ until analysis. The brain samples were weighed, homogenized in 10

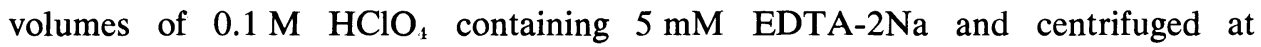
$20000 \times \mathrm{g}$ and $4^{\circ} \mathrm{C}$. Concentrations of TRP, serotonin and 5-HIAA in the supernatant fluid were determined by high performance liquid chromatography with continuous fluorometric monitoring. They were measured by elution in a Yanapak ODS-T column with $12 \% \mathrm{MeOH}-0.1 \mathrm{M} \mathrm{NaH}_{2} \mathrm{PO}_{4}$ (pH 3.5) and the formation of OPA derivatives. ${ }^{9)}$

\section{Statistical Analysis}

Statistical analysis was made by two-tailed or paired t-test.

\section{RESULTS}

1) Changes in EEG-spectral profile, body temperature and behavior after swimming.

It was found by power spectral analysis of EEG in the parieto-occipital cortex that the frequency of the main peak in the EEG power spectra was lowered to $4 \mathrm{~Hz}$ after swimming, while the frequency of the main peak of the arousal EEG ranged from 6 to $8 \mathrm{~Hz}$ during the control period. However, the lowered frequency of the main peak returned to normal within 2 hours. The rectal temperature of the six rats was $29.4 \pm 0.4^{\circ}$ immediately after swimming, while the rectal temperature of the control rats was $37.8 \pm 0.2^{\circ} \mathrm{C}$. The difference in the rectal temperature 


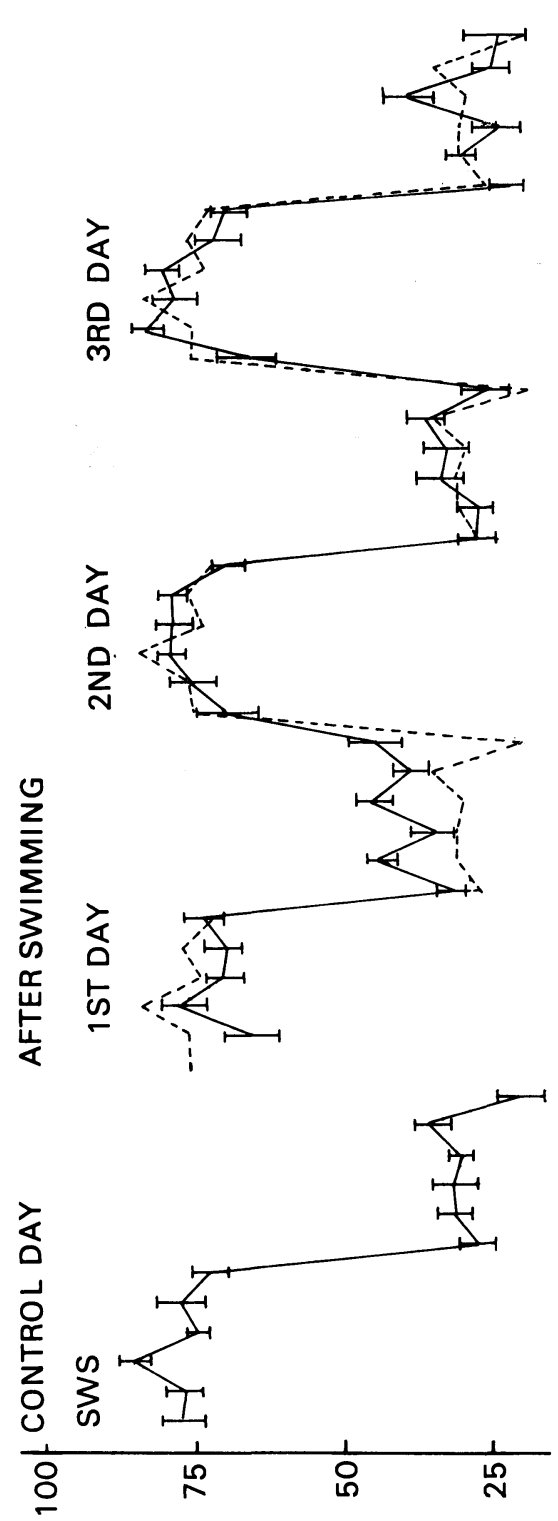

(sınouz/u!m) SMS

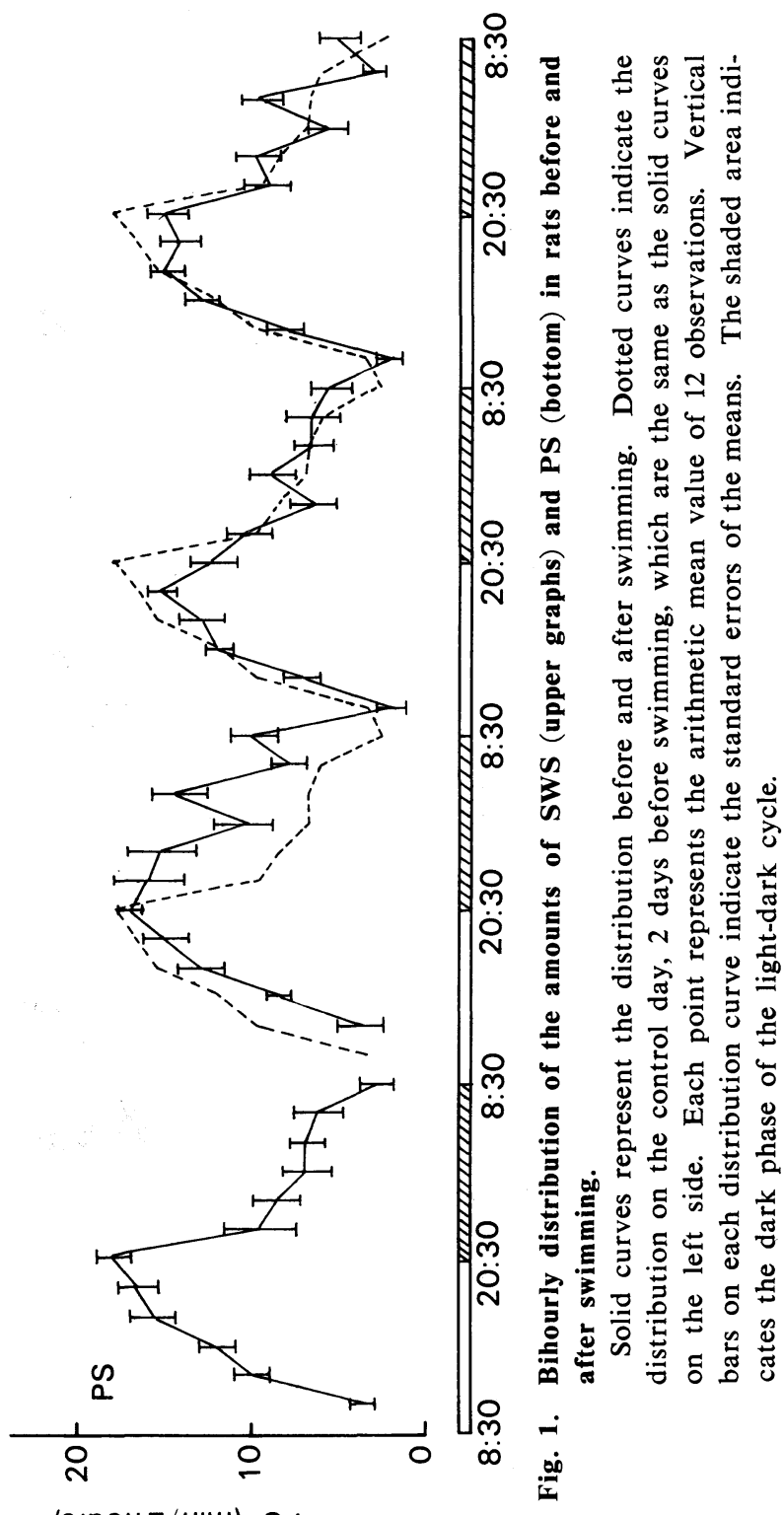

(sınouZ/u!w) Sd 
between the two groups was not significant about 2 hours later. The rats loaded with swimming did not show any ambulatory activity, although their posture and righting reflex were normal.

\section{2) Sleep-waking patterns}

Sleep patterns of rats before and after the swimming are illustrated in Fig. 1 by bihourly distribution of SWS and PS. In the control period, the amount of SWS was consistently greater in the light period than in the dark period. The amount of PS was relatively low in the dark and after the illumination gradually increased to a peak around 18:00. These baseline circadian rhythms of SWS and PS are in good accordance with those reported previously. ${ }^{7)}$

Because of the abnormal EEG patterns for 2 hours after swimming, this period of time was not divided into sleep and wakefulness. In the later part of the light period (10:30-20:30), the SWS level was slightly lower than in the same time period of the control day $(\mathrm{p}<0.01)$. The PS level showed a similar tendency. In the dark period, however, the SWS level was significantly greater than the control level ( $p<0.05$ for the first half of the dark period) and PS also showed an increased level $(\mathrm{p}<0.01$ for the same period, Table 1$)$. It was found that SWS was increased only in the dark period. These differences were not observed on the second day after the exercise, except for the latter half of the light period when PS was still reduced. Therefore, it was thought that the brain indoles should be measured in the post-exercise dark period, to obtain some information about the relationship between exercise-induced increase of SWS and brain serotonin metabolism.

Table 1. Changes of the amounts of SWS and PS in rats after swimming

\begin{tabular}{|c|c|c|c|c|c|c|c|c|}
\hline \multirow{3}{*}{ Time } & \multirow{2}{*}{\multicolumn{2}{|c|}{$\begin{array}{l}\text { Control period } \\
2 \text { days before } \\
\text { swimming }\end{array}$}} & \multicolumn{6}{|c|}{ After swimming } \\
\hline & & & \multicolumn{2}{|l|}{ 1st day } & \multicolumn{2}{|l|}{ 2nd day } & \multicolumn{2}{|c|}{ 3rd day } \\
\hline & $(\min )$ & $(\%)$ & $(\min )$ & $(\%)$ & $(\min )$ & $(\%)$ & $(\min )$ & $(\%)$ \\
\hline \multicolumn{9}{|l|}{ SWS } \\
\hline $\left.10: 30-14: 30^{a}\right)$ & $160.7 \pm 3.7 b)$ & $67.0^{\mathrm{c})}$ & $144.5 \pm 5.1^{* *}$ & 60.2 & $158.0 \pm 4.0$ & 65.8 & $170.2 \pm 5.9$ & 70.9 \\
\hline $14: 30-20: 30$ & $226.6 \pm 5.3$ & 62.9 & $216.7 \pm 6.1^{* *}$ & 60.2 & $231.3 \pm 5.5$ & 64.2 & $225.8 \pm 5.7$ & 62.7 \\
\hline $20: 30-2: 30$ & $92.0 \pm 8.7$ & 25.6 & $114.2 \pm 5.6^{*}$ & 31.7 & $93.0 \pm 6.7$ & 25.8 & $81.6 \pm 4.8$ & 22.7 \\
\hline $2: 30-8: 30$ & $88.8 \pm 6.4$ & 24.7 & $132.7 \pm 7.0^{* *}$ & 36.9 & $99.1 \pm 8.3$ & 27.5 & $94.0 \pm 7.4$ & 26.1 \\
\hline \multicolumn{9}{|l|}{ PS } \\
\hline $10: 30-14: 30$ & $20.4 \pm 1.4$ & 8.5 & $12.6 \pm 1.7^{* *}$ & 5.2 & $19.3 \pm 1.7$ & 8.0 & $22.8 \pm 2.6$ & 9.5 \\
\hline $14: 30-20: 30$ & $50.3 \pm 2.7$ & 14.0 & $45.0 \pm 2.0$ & 12.5 & $40.7 \pm 2.3^{* *}$ & 11.3 & $44.5 \pm 2.0$ & 12.4 \\
\hline $20: 30-2: 30$ & $25 \cdot 6 \pm 2.4$ & 7.1 & $42.3 \pm 3.3^{* *}$ & 11.7 & $25.9 \pm 1.9$ & 7.2 & $25.5 \pm 1.7$ & 7.1 \\
\hline $2: 30-8: 20$ & $16.5 \pm 2.4$ & 4.6 & $32.2 \pm 2.9 * *$ & 8.9 & $18.9 \pm 2.2$ & 5.2 & $18.4 \pm 2.0$ & 5.1 \\
\hline
\end{tabular}

a) Clock time.

b) The mean and standard error of 12 observations.

c) The time spent in SWS or PS divided by the total recording time (\%).

$* \mathrm{p}<0.05$, ** $\mathrm{p}<0.01$ (paired $\mathrm{t}$-test, compared with the control periods). 
Table 2. Brain TRP, serotonin and 5-HIAA levels in rats 15 hours after swimming

\begin{tabular}{|c|c|c|c|c|c|c|}
\hline \multirow{2}{*}{ Group } & \multicolumn{2}{|c|}{$\begin{array}{c}\text { TRP } \\
(\mathrm{nmol} / \mathrm{g} \text { wet tissue })\end{array}$} & \multicolumn{2}{|c|}{$\begin{array}{c}\text { Serotonin } \\
\text { (nmol/g wet tissue) }\end{array}$} & \multicolumn{2}{|c|}{$\begin{array}{c}\text { 5-HIAA } \\
(\mathrm{nmol}) / \mathrm{g} \text { wet tissue })\end{array}$} \\
\hline & Control & Swimming & Control & Swimming & Control & Swimming \\
\hline \multicolumn{7}{|l|}{ Brain region } \\
\hline Midbrain & $19.1 \pm 0.8$ & $22.0 \pm 0.8^{*}$ & $4.04 \pm 0.12$ & $4.63 \pm 0.72$ & $3.78 \pm 0.06$ & $4.41 \pm 0.15^{* *}$ \\
\hline Cerebral cortex & $18.9 \pm 0.6$ & $20.2 \pm 0.5$ & $2.40 \pm 0.20$ & $2.36 \pm 0.14$ & $1.62 \pm 0.04$ & $1.88 \pm 0.06^{* *}$ \\
\hline Hypothalamus & $19.2 \pm 0.5$ & $20.0 \pm 0.8$ & $4.24 \pm 0.15$ & $4.06 \pm 0.11$ & $3.06 \pm 0.07$ & $3.25 \pm 0.18$ \\
\hline Hippocampus & $19.1 \pm 0.6$ & $20.3 \pm 1.0$ & $1.98 \pm 0.06$ & $1.86 \pm 0.16$ & $2.04 \pm 0.05$ & $2.21 \pm 0.09$ \\
\hline Pons-medulla & $19.7 \pm 1.1$ & $22.4 \pm 1.0$ & $3.09 \pm 0.14$ & $2.86 \pm 0.16$ & $2.11 \pm 0.17$ & $2.09 \pm 0.19$ \\
\hline
\end{tabular}

The rats were kept in water of 25 to $28^{\circ} \mathrm{C}$ during the last 3.5 hours of the dark period, and 15 hours later they were killed.

Each value represents the mean and standard error of 6 animals.

* $\mathrm{p}<0.05$, ** $\mathrm{p}<0.01$ (two-tailed t-test).

\section{3) Brain TRP, serotonin and 5-HIAA levels.}

Table 2 shows TRP, serotonin and 5-HIAA levels in the 5 brain regions of the control and the exercised rats 15 hours after the swimming. In the midbrain, TRP and 5-HIAA levels were significantly higher $(\mathrm{p}<0.05$ and $\mathrm{p}<0.01$, respectively) in the exercised rats than in the control ones. The 5-HIAA content of the cerebral cortex was also significantly higher $(p<0.01)$ in the exercised animals than in the control. The serotonin levels in the two brain regions seemed unaffected by the exercise. In the other brain regions, there was no significant difference in the indole levels between the two groups.

\section{4) Histological examination}

Gross observation revealed that petechiae were present in the glandular stomach in four out of the six rats loaded with swimming. Slight ulcers and hemorrhages of the stomach mucosa were observed microscopically in two exercised rats.

\section{Discussion}

It has been demonstrated by animal experiments that the amount of SWS is increased after physical exercise. Hobson ${ }^{2)}$ found an increase in the amount of SWS in cats immediately after the cessation of moderate treadmill exercise. $\mathrm{K}$. Matsumoto et al. ${ }^{3)}$ reported that when rats were loaded with swimming for 3 hours in the early part of the light period, a significant increase in the amount of deep SWS was found during the next several hours of the light period. In the present study, however, there was a decrease in the amount of SWS in the first 12 hours of the light period after the exercise, and a marked increase of SWS was observed in the following dark period in rats. The discrepancy might be partially attributable to further division of SWS into deep and light ones. Since the two types of SWS were not measured separately in the present study, it is not clear whether deep 
SWS increases in the light period after swimming exercise in rats.

Another reason for delayed appearance of the exercise-induced increase in SWS amount might be due to the type of exercise used. The present study dealt with an exhaustive and stressful swimming for 3.5 hours at 25 to $28^{\circ} \mathrm{C}$. Immediately after the exercise, the rectal temperature of all rats was reduced and slight ulcers and hemorrhages of stomach mucosa were observed in several rats. In the experiment by $\mathrm{K}$. Matsumoto et al.,3) the swimming in water of $30^{\circ} \mathrm{C}$ was loaded for 3 hours. Baekeland and Lasky ${ }^{5}$ ) found that evening exercise in man produced an increase in the amount of wakefulness and no increase in delta sleep during subsequent night sleep, while a marked increase of delta sleep was found after afternoon exercise. They suggested that evening exercise may exert a stress effect opposing a general tendency of exercise to increase delta sleep requirements. It seems reasonable to consider that sleep disturbance could be produced as a result of stress reactions accompanied by the present swimming exercise.

To clarify the possible role of central serotonergic neurons involved in the mechanisms of SWS, brain TRP, serotonin and 5-HIAA were measured at the time of appearance of SWS increase after exercise. The concentrations of TRP and 5-HIAA in the midbrain were significantly higher in the exercised rats at the time of increased SWS in the dark period. The cortical levels of 5-HIAA and TRP were also enhanced at the same time. The serotonin levels in these regions were not affected. It was reported that brain serotonin synthesis is influenced by the supply of TRP to the brain. ${ }^{10)}$ The present results indicate that degradation of serotonin to 5-HIAA and synthesis of serotonin from TRP were enhanced in the first dark period after swimming when the amount of SWS was increased. Additionally, the supply of TRP to the brain regions seemed to be increased in the post-exercise dark period.

Involvement of central serotonergic neurons in sleep mechanisms, especially in SWS, was already suggested by Jouvet ${ }^{6)}$ who demonstrated insomnia after surgical destruction of the raphe nuclei and pharmacological manipulation of the serotonergic neurons in cats. Radulovacki and his coworkers ${ }^{11,12)}$ demonstrated that levels of serotonin and 5-HIAA in the hippocampus were higher during SWS than the indole levels during waking in cats. Ogasahara et al. ${ }^{13)}$ also reported an enhanced turnover rate of serotonin in the hippocampus during SWS as compared with the rates during waking in rats. Enhanced turnover rates of brain serotonin during rebound sleep were reported by Toru et al. ${ }^{14)}$ who showed increased 5-HIAA concentration in the dorsal raphe nucleus area and thalamus immediately after 24-hour sleep deprivation in rats and when the rats had a 3-or 30-min rebound sleep. On the other hand, TRP, which is a precursor in serotonin synthesis, has been known to be hypnotic. ${ }^{15)}$ Wojcik et al. ${ }^{16)}$ confirmed that SWS latency was reduced by intraperitoneal administration of L-TRP in rats, and found that the 5-HIAA level was increased in the pons-medulla and cortex at almost the same time as the time of the first appearance of SWS episode after 
the injection of L-TRP. They suggested that the sleep-inducing effect of L-TRP may be mediated through brainstem serotonergic neurons.

In consideration of these facts, an increase in the amount of sleep, especially in SWS, seems to be associated with increased supply of TRP to the brain and an enhanced turnover rate of brain serotonin. It may be concluded that the increased amount of SWS after swimming exercise is attributable to enhanced activity of ascending serotonergic neurons which innervate the midbrain and the cerebral cortex.

The level of PS in the dark period was significantly increased after swimming in the present study. However, the role of serotonergic neurons in the PS mechanisms has not been clarified yet. Further investigation will be necessary in the respect of the increased PS.

\section{ACKNOWLEDGEMENT}

The authors are grateful to Prof. S. Torii, Department of Physiology, Toho University School of Medicine, for his kind advice.

\section{REFERENCES}

1) Matsumoto, J., Nishisho, T., Suto, T., Sadahiro, T. and Miyoshi, M. (1968). Influence of fatigue on sleep, Nature, 218, 177.

2) Hobson, J. A. (1968). Sleep after exercise, Science, 162, 1503.

3) Matsumoto, K., Matsui, T. and Kawamori, M. (1982). The effects of forced exercise on the sleep of rats, Jpn. J. Hyg., 37, 505.

4) Shapiro, C. M., Bortz, R., Mitchell, D., Bartel, P. and Jooste, P. (1981). Slow-wave sleep: a recovery period after exercise, Science, 214, 1253.

5) Baekeland, F. and Lasky, R. (1966). Exercise and sleep patterns in college athletes, Percep. Mot. Skills, 23, 1203.

6) Jouvet, M. (1972). The role of monoamines and acetylcholine-containing neurons in the regulation of sleep-waking cycle, Ergeb. Physiol., 64, 166.

7) Arito, H., Sudo, A., Hara, N., Nakagaki, K. and Torii, S. (1982). Changes in circadian sleep-waking rhythms of rats following administration of methylmercury chloride, Ind. Health, 20, 55.

8) Glowinski, J. and Iversen, L. L. (1966). Regional studies of catecholamines in the rat brain. I. The disposition of ${ }^{3} \mathrm{H}$-norepinephrine, ${ }^{3} \mathrm{H}$-dopamine and ${ }^{3} \mathrm{H}$-dopa in various regions of the brain, J. Neurochem., 13, 655.

9) Kojima-Sudo, A. (1977). Analysis of serotonin and histamine by high-speed liquid chromotograph and autoanalyzer, Ind. Health, 15, 109.

10) Gessa, G. L. and Tagliamonte, A. (1974). Possible role of free serum tryptophan in the control of brain tryptophan level and serotonin synthesis, Adv. Biochem. Psychopharmacol., 11, 119.

11) Buckingham, R. L. and Radulovacki, M. (1975). 5-Hydroxyindoleacetic acid in cerebrospinal fluid: an indicator of slow-wave sleep, Brain Res., 99, 440.

12) Kovacevic, R. and Radulovacki, M. (1976). Monoamine changes in the brain of cats during slow-wave sleep, Science, 193, 1025.

13) Ogasahara, S., Taguchi, Y. and Wada, H. (1980). Changes in serotonin in rat brain 
during slow-wave sleep and paradoxical sleep: application of the microwave fixation method to sleep research, Brain Res., 189, 570.

14) Toru, M., Mitsushio, H., Mataga, N., Takashima, M. and Arito, H. (1984). Increased brain serotonin metabolism during rebound sleep in sleep-deprived rats, Pharmacol. Biochem. Behav., 20, 757.

15) Hartmann, E. and Chung, R. (1972). Sleep-inducing effects of L-tryptophan, J. Pharm. Pharmcol., 24, 252.

16) Wojcik, W. J., Fornal, C. and Radulovacki, M. (1980). Effect of tryptophan on sleep in the rat, Neuropharmacology, 19, 163. 